\title{
Enhanced expression of genes in the brains of larvae of Mamestra brassicae (Lepidoptera: Noctuidae) exposed to short daylength or fed Dopa
}

\author{
Masahide URYU, Yohsuke NINOMIYA, TAKeshi YOKOI, SeiJI TSUZUKI and YoIChI HAYAKAWA* \\ Institute of Low Temperature Science, Hokkaido University, Sapporo 060-0819, Japan
}

Key words. Diapause, Mamestra brassicae, cabbage armyworm, brain, short day, Dopa, dopamine, gene expression, receptor for activated C-kinase

\begin{abstract}
The cabbage armyworm, Mamestra brassicae, enters diapause in the early pupal stage. Pupal diapause is induced by rearing the larvae under short day lengths. We previously demonstrated that feeding Dopa during last larval instar induces pupal diapause even under long day lengths. In order to elucidate the mechanism by which pupal diapause is induced after experiencing short day lengths or fed Dopa under long day lengths, we analyzed gene expression in the brain of $M$. brassicae larvae under both of these conditions using a subtractive hybridization technique. After the secondary screen, 49 clones and 28 clones were identified as short day length or Dopa-feeding specific clones, respectively. All of these genes were sequenced and, using the base sequences of these clones, primers were synthesized. To confirm the genes enhanced specifically by these conditions, quantitative real-time PCR was carried out. This quantitative PCR analysis identified 15 and 1 clone whose expression was enhanced by the short day length conditions or Dopa-feeding, respectively. Among these clones, the gene with a high level of identity to receptor for activated protein kinase C (RACK) from Heliothis virescens is the most dramatically up-regulated under both conditions.
\end{abstract}

\section{INTRODUCTION}

Insect diapause is characterized by developmental or reproductive arrest and a decrease in metabolic rate. Diapause has evolved in most insect species to ensure survival in unfavorable periods and to synchronize the growth rate of the population. The diapause program is usually triggered by environmental conditions such as temperature and photoperiod, as perceived during a developmentally-specific sensitive stage, which is more or less distant from the stage where diapause is manifested (Denlinger, 1985). The photoperiod can be used as a reliable seasonal cue in comparison to temperature, because it is seasonally highly predictable. That is the reason why so many insect species use photoperiod as the cue for diapause induction.

The cabbage armyworm, Mamestra brassicae, has the capacity to enter diapause in the pupal stage: Larvae reared under short day lengths $(\mathrm{SD})$ at $22^{\circ} \mathrm{C}$ metamorphose into diapausing pupae, whereas those maintained under long day lengths (LD) at $22^{\circ} \mathrm{C}$ develop into nondiapausing pupae (Kimura \& Masaki, 1992, 1993). Prior studies indicate that dopamine concentrations are elevated in the hemolymph and central nervous system of diapause-destined $M$. brassicae pupae reared under SD (Noguchi \& Hayakawa, 1997). Further, more than $50 \%$ of the pupae fed Dopa during their last larval instar stage entered a diapause-like state even when maintained under $\mathrm{LD}$ at $22^{\circ} \mathrm{C}$. Dopamine concentrations were higher in the hemolymph and central nervous system of the Dopa-fed insects than in non-diapausing control insects around the time of pupal ecdysis (Noguchi \& Hayakawa, 1997). These results indicate that the increase in dopamine con- centrations in hemolymph and nervous system should contribute to the induction of diapause. However, even if dopamine in these tissues is closely related to the induction of diapause, the molecular mechanism underlying this phenomenon remains unknown. Further, we do not know how similar or different are the effects of exposure to SD and feeding Dopa to larvae. However, we know that these two stimuli affect the larval brain during similar stages and then induce diapause.

In order to address these questions, it is necessary to examine in more detail the molecular events in the brain of the larvae that are exposed to these stimuli. The present study was conducted to search for genes whose expression is elevated in the brain of larvae exposed to SD and/or fed Dopa.

\section{MATERIALS AND METHODS}

\section{Animals}

Larvae of the cabbage armyworm $M$. brassicae were reared on an artificial diet at $22^{\circ} \mathrm{C} \pm 1{ }^{\circ} \mathrm{C}$ and a photoperiod of $14 \mathrm{~h}$ light : $10 \mathrm{~h}$ dark (long day length, LD) or $10 \mathrm{~h}$ light : $14 \mathrm{~h}$ dark (short day length, SD) (Hayakawa \& Ohnishi, 1998). More than $80 \%$ of larvae reared under the LD condition metamorphosed from pupae to adults within 2 weeks of pupation. Therefore, those pupae were defined as non-diapausing. Larvae reared under the SD condition showed no signs of adult development for 8 weeks after pupation and were regarded as diapause pupae. The cabbage armyworms maintained in this laboratory do not show a typical aestival-diapause by rearing under the LD condition. Fourth and fifth instar larvae undergoing ecdysis between 4 and $4.5 \mathrm{~h}$ after lights on were designated as Day 0 penultimate and last instar larvae, respectively.

To study the effect of a number of SD cycles on diapause induction, larvae were reared under the LD condition until the

\footnotetext{
* Corresponding author. Tel: 011-706-6880; fax: 011-706-7142; e-mail: hayakawa@lowtem.hokudai.ac.jp
} 
A
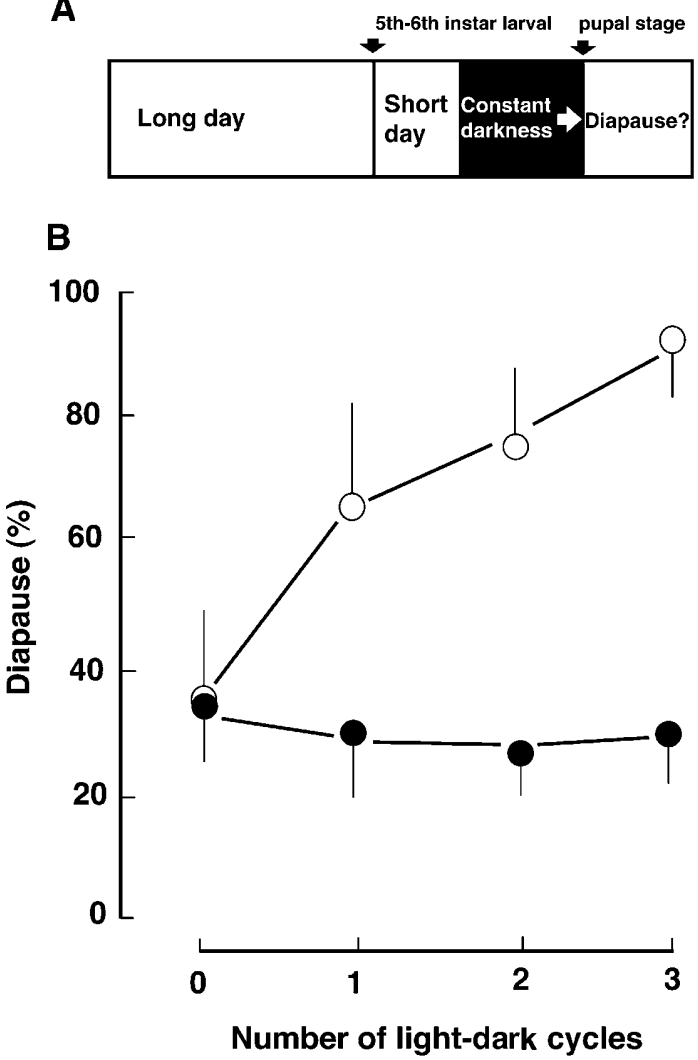

Fig. 1. Induction of pupal diapause in response to short-day cycles. A - experimental procedure: $M$. brassicae larvae reared under LD until the 4th instar were exposed to SD for a variable number of days and then kept in darkness until pupation; B the diapause incidence induced by exposure to SD $(O)$. The diapause incidence in control larvae kept in LD condition before transfer to constant darkness $(\bullet)$. Pupae which showed no signs of adult development after 8 weeks were defined as in diapause. Each point represents a mean \pm S.D. based on from $13-15$ independent determinations.

4th instar, then exposed to the SD condition for a variable number of days, and then kept in constant darkness until pupation. For the subtractive hybridization experiments, test larvae were reared under the SD condition for 3 days after 5th larval ecdysis (SD-treated) or larvae were fed an artificial diet containing 1\%(w/w) Dopa for 3 days after 5 th larval ecdysis (under the LD condition) (DP-treated). Control larvae were reared under the LD condition throughout their development.

\section{Subtraction of cDNAs}

Total RNAs were isolated from test (SD- and Dopa-treated) and control (LD) larvae using the method of Chomczynski \& Sacchi (1987) and enriched for poly(A) ${ }^{+}$RNA using the QuickPrep $^{\text {TM }}$ Micro mRNA Purification Kit (Amersham Bioscience Co.). The PCR-Select cDNA Subtraction Kit (BD Bioscience Clontech) was used to isolate transcripts strongly expressed under diapause-inducible conditions, basically according to the manufacturer's instructions. Tester cDNAs were created from poly(A) ${ }^{+}$RNA prepared from SD- or DP-treated larvae. Driver cDNAs were simply prepared from larvae reared under the LD condition. After hybridization of tester and driver cDNAs, subtraction of tester unique DNAs was performed using the method based on selective amplification of differentially expressed sequences. The pool of subtracted cDNAs was cloned into a
TA-cloning plasmid vector (pGEM-T, Promega), and all of the clones obtained were subjected to further screening. After confirming the candidate genes with dot hybridization, nucleotide sequences of the isolated clones were determined with an automatic DNA sequencer, ABI Prism model 377 (Applied Biosystems) (Noguchi \& Hayakawa, 2001).

\section{Quantitative Real-time PCR (qPCR)}

qPCR was performed by using the Light Cycler and the Fast Start DNA Master SYBR Green I kit (Roche Diagnostics) according to the procedure of Menssen \& Hermeking (2002). For qPCR of RACK, primer pairs for RACK and B-tublin (as an external standard) are as follows:

RC-1, 5'- TTGGTTTCGTCTCTGGTCAACTTC;

RC-2, 5'-GTTGGTGCTTAGCCGCTCAGCAAG;

Tb-1, 5'-AAGAGCTCTGGAGCCMGGYACSATGGACTCKGT;

Tb-2, 5'-AAGAGCTCGTCTTCACGTTGTTGGGGATCCA.

The reverse transcription was performed on total RNA after DNase I treatment (DNA-free, AMBION) using ReverTra Ace- $\alpha$-cDNA synthesis kit (Toyobo, Japan). The generation of specific PCR products was confirmed by melting curve analysis and gel electrophoresis. Each primer pair was tested with a logarithmic dilution of a cDNA mix to generate a linear standard curve (crossing point CP plotted vs. log of template concentration), which was used to calculate the primer pair efficiency.

\section{Rapid amplification of RACK cDNA ends (5'RACE and 3'RACE)}

Total RNA was isolated from larvae reared under the SD condition. Adapter-ligated double-strand cDNAs were synthesized using a 5'RACE and 3'RACE Rapid Amplification system (Roche Diagnostics) according to the manufacturer's instructions. PCR was carried out in $20 \mu \mathrm{l}$ of $10 \mathrm{mM}$ Tris- $\mathrm{HCl}$ (pH 8.3) containing $50 \mathrm{mM} \mathrm{KCl}, 2 \mathrm{mM} \mathrm{MgCl}, 0.01 \%(\mathrm{w} / \mathrm{v})$ gelatin, 0.25 $\mathrm{mM}$ each of dNTPs, $200 \mathrm{nM}$ each set of primers, $0.2 \mu 1$ of synthesized cDNA solution and $0.5 \mathrm{U}$ of Taq (Takara, Japan) as described previously (Hayakawa et al., 1995). The 5'RACE and 3'RACE products were subcloned into TA-cloning plasmid vector (pGEM-T, Promega) and sequenced in both directions by a Taq dye primer cycle sequencing kit (Perkin-Elmer) using an automatic DNA sequencer (model 377, PE Applied Biosystems) (Hayakawa \& Noguchi, 1998).

\section{In situ hybridization}

Brains from Day 2 penultimate instar larvae were fixed in $4.0 \%$ paraformaldehyde and hybridized as described previously (Hayakawa et al., 1998; Tanaka et al., 2002). The hybridization probe was antisense RACK mRNA labeled with digoxygenin, and the sense mRNA was used as a negative control probe (Tauz \& Pfeifle, 1989).

Abbreviations. Dopa - 3,4-dihydroxy-L-phenylalanine; PKC - protein kinase C; RACK - receptor for activated protein kinase $\mathrm{C}$.

\section{RESULTS}

\section{Diapause induction under short day length}

To elucidate the number of short-day cycles required to induce pupal diapause in the cabbage armyworm Mamestra brassicae, larvae reared under long day length (LD) were transferred to short day length (SD) from penultimate instar stage. The incidence of diapause increased with increasing number of SD cycles from 0 to 3 (Fig. 1). When larvae were exposed to SD for 3 days, the incidence of diapause is over $80 \%$. 


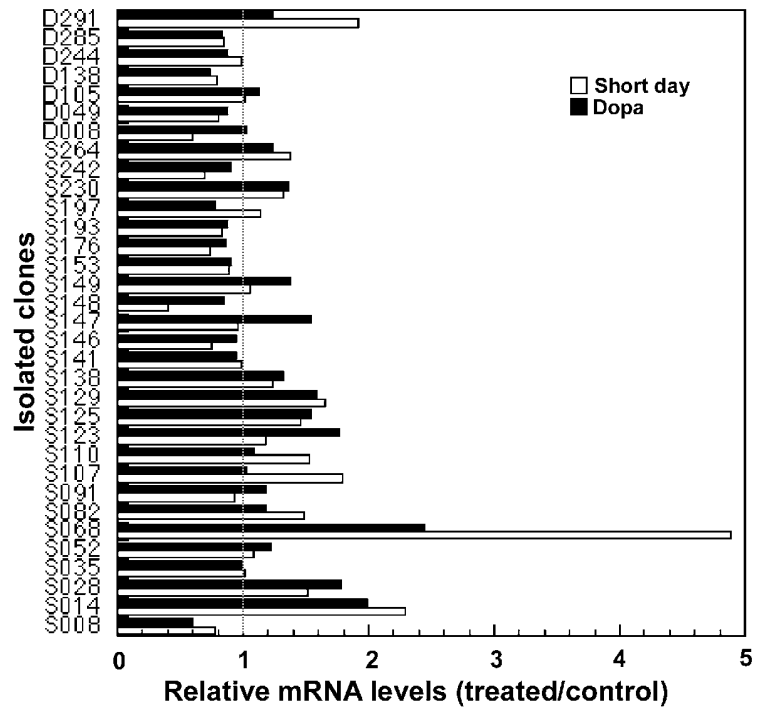

Fig. 2. Relative levels of mRNAs of genes isolated by subtractive hybridization. Each gene mRNA was measured in SD or Dopa fed larvae using quantitative real-time PCR. When there is no difference in mRNA levels between test (SD or Dopa fed) and control (LD condition), the relative value is 1 . Each bar is the mean of two independent determinations. Closed bar, short day length; open bar, fed Dopa.

Prior studies demonstrated that feeding artificial diet containing 1\% Dopa induced diapause-like state in over $50 \%$ of the pupae even under LD (Noguchi \& Hayakawa, 1997). Thus, subtractive hybridization was conducted to identify the genes whose expression is enhanced in the brains of cabbage armyworm larvae that experienced three cycles of SD or were fed Dopa (DP) for 3 days.

\section{Identification of genes related to diapause-destination}

By initial screening, each of 300 clones were selected as up-regulated genes in SD or DP conditions as compared to those in LD. A second round of screening was performed using dot hybridization for 600 clones using cDNAs prepared from LD-, SD- and DP-conditioned larvae, and clones which hybridized strongly to SD- or DPlarval cDNAs in comparison to LD-larval cDNAs were selected. The overall protocol is summarized in Table 1, which reveals that 49 and 28 clones were identified as upregulated specifically for SD- and DP-conditions, respectively. All of the clones were sequenced, and partial sequences of 33 clones were successfully obtained. Based on the sequence data of these clones, specific primers were synthesized for PCR. Quantitative real-time RT-PCR revealed that one clone, S068, is the most up-

TABLE 1. Protocol for differential screening.

\begin{tabular}{lcc}
\hline & \multicolumn{2}{c}{ Numbers of isolated clones } \\
\cline { 2 - 3 } & $(\mathrm{SD})-(\mathrm{LD})$ & $(\mathrm{Dopa})-(\mathrm{LD})$ \\
\hline 1st subtration & 300 & 300 \\
Dot blotting & 49 & 28 \\
Sequencing & 49 & 28 \\
Real time PCR & 26 & 7 \\
Up-regulated & 15 & 1 \\
\hline
\end{tabular}

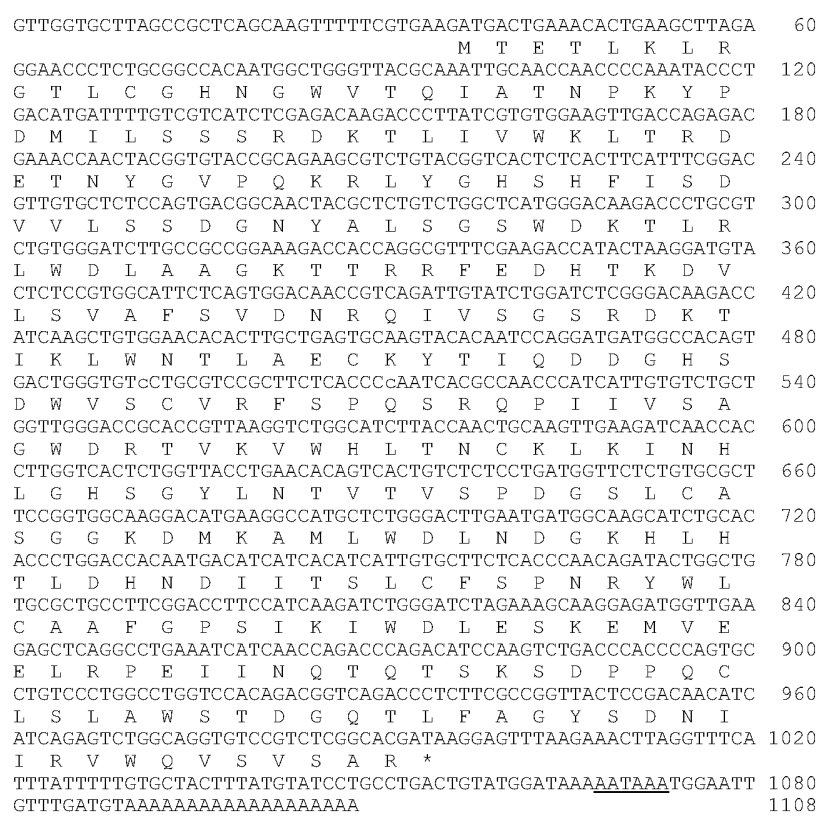

Fig. 3. Nucleotide and predicted amino acid sequence of $M$. brassicae RACK (receptor for activated C-kinase) cDNA. The clone has an open reading frame of 319 amino acids. The putative polyadenylation signal is underlined.

regulated both in SD- and DP-conditions among the above putative up-regulated clones (Fig. 2).

\section{The cDNA structure of S068 clone}

To determine the structure of the S068 cDNA, 5'- and 3'- RACE were performed and the full sequence was analyzed in both directions. The predicted primary structure indicates the presence of a protein of 319 amino acids (Fig. 3). The sequence similarity to the S068 protein revealed significant homology with receptor for activated protein kinase $\mathrm{C}$ (RACK) reported for many species of animals including vertebrates and invertebrates (Table 2). Therefore, we concluded that S068 is a Mamestra brassicae RACK gene.

\section{Expression of $M$. brassicae RACK gene under LD and SD conditions}

To examine whether the expression of $M$. brassicae RACK gene is enhanced in the brains of SD- and DPconditioned larvae, RACK mRNAs in larvae reared under LD-, SD- and DP-conditions were measured by quantitative real-time PCR (Fig. 4). The RACK mRNA increased in DP (Dopa-fed) larval brain within one day after feeding Dopa and maintained the increased levels at least for 3 days. The RACK gene expression was also higher in the brains of larvae under SD-condition than LDcondition, while obvious increase of the RACK mRNA was observed 2 days after transfer from LD to SD.

The RACK gene expression in the brain was further examined by in situ hybridization on paraformaldehydefixed, paraffin-embedded sections of brain from Day 3 penultimate instar larvae (Fig. 5). The expression of RACK mRNA was mainly detected in several cells around the medial protocerebral neuropile both under SDand LD-conditions. However, the expression is more 


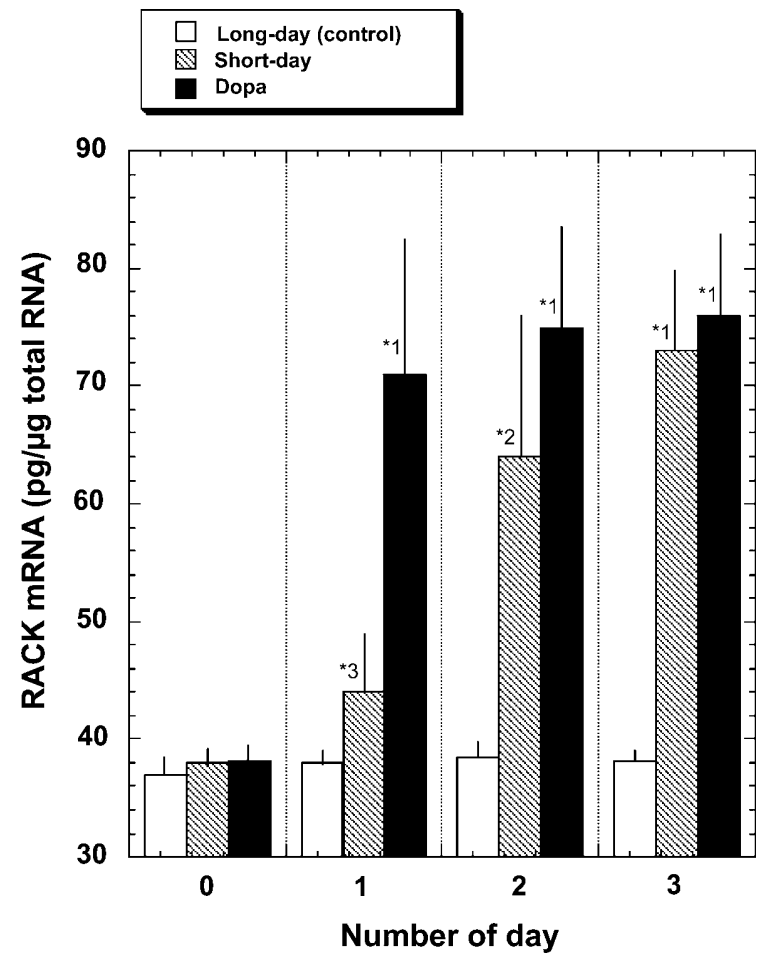

Fig. 4. Mean RACK mRNA levels of $M$. brassicae larvae exposed to SD and fed Dopa. Larvae reared under LD until 4th instar larvae were exposed to SD or fed Dopa for the indicated number of days and RACK mRNA was measured using quantitative real-time PCR. ${ }^{*} 1$, significantly different from control LD-conditioned larval value $(\mathrm{P}<0.001$ : Student's t-test). *2, significantly different from control value $(\mathrm{P}<0.01$ : Student's test). $* 3$, not significantly different from control value $(\mathrm{P}>$ $0.05)$. Each point represents the mean \pm S.D. of 3 independent determinations. $\triangle$, Dopa; $O$, short day length; $\bullet$, long day length.

obvious in SD-conditioned larval brains than in LDconditioned, and no positive signal could be detected in the control brain using the sense probe.

\section{DISCUSSION}

Prior studies demonstrated that the dopamine content in the central nervous tissues is several times higher in diapause-destined than in non-diapause-destined M. brassicae around pupation. It is likely that dopamine inhibits the activity of neurosecretions, such as PTTH, a requisite for adult development; thereby, the diapause-destined pupae with a high level of dopamine in their brains do not initiate adult development and enter diapause (Noguchi \& Hayakawa, 1997). Although detailed analysis of the dopamine effect on PTTH release from the brain is certainly required to substantiate this prediction, we believe that this in broad outline is the mechanism by which diapause is induced during pupation.

Another important question is how the SD signal is transduced into a diapause inducing program in the brains of $M$. brassicae larvae. We thus conducted the subtractive hybridization for identifying genes that are expressed strongly under the SD condition as compared with the LD condition. Among several putative SD-up-regulated genes
TABLe 2. Sequence identities and similarities between $M$. brassicae RACK and RACKs of other species.

\begin{tabular}{lcc}
\hline & Identity (\%) & Similarity (\%) \\
\hline $\begin{array}{l}\text { Heliothis virescens } \\
\text { (AF368031) }\end{array}$ & 97 & 97 \\
$\begin{array}{l}\text { Drosophila melanogaster } \\
\text { (U96491) }\end{array}$ & 84 & 90 \\
$\begin{array}{l}\text { Rattus norvegicus } \\
\text { (A36986) }\end{array}$ & 77 & 87 \\
$\begin{array}{l}\text { Brachydanio rerio } \\
\text { (AF025330) }\end{array}$ & 77 & 87 \\
$\begin{array}{l}\text { Oreochromis niloticus } \\
\text { (AF025331) }\end{array}$ & 76 & 86 \\
$\begin{array}{l}\text { Homo sapiens } \\
\text { (M24194) }\end{array}$ & 76 & 86 \\
$\begin{array}{l}\text { Mus musculus } \\
\text { (D29802) }\end{array}$ & 76 & 86 \\
$\begin{array}{l}\text { Xenopus laevis } \\
\text { (AF105259) }\end{array}$ & 76 & 86 \\
$\begin{array}{l}\text { Biomphalaria glabrata } \\
\text { (U49437) }\end{array}$ & 75 & 85 \\
$\begin{array}{l}\text { Hydra attenuata } \\
\text { X97800) }\end{array}$ & 69 & 81 \\
$\begin{array}{l}\text { Euprymna scolopes } \\
\text { (AF124742) }\end{array}$ & 73 & \\
$\begin{array}{l}\text { Caenorhabditis elegans } \\
\text { (Z69664) }\end{array}$ & 71 & \\
$\begin{array}{l}\text { Neurospora crassa } \\
\text { (X81875) }\end{array}$ & 76 & \\
\hline & 76 & \\
\hline
\end{tabular}

identified by this method, receptor for activated protein kinase C (RACK) was the most up-regulated gene under the SD-condition (Fig. 2). The enhanced expression of the RACK gene was also observed in larvae fed Dopa (Figs 2, 4). The members of the Protein kinase C (PKC) family transduce a multitude of signals that regulate various cellular functions leading to cell survival or to cell death. $\mathrm{PKC}$ is considered to act as the intracellular receptor for the second messenger diacylglycerol (Nishizuka, 1984, 1988). Various environmental signals stimulate the production of diacylglycerol through the activation of phospholipase $\mathrm{C}$ and as such PKC is likely to have a key role in the response to these signals (Meldrum et al., 1991). Activation of PKC leads to translocation of the enzyme from the cytosol to the particulate fraction (Kraft \& Anderson, 1983). It is thought that PKC translocates to subcellular sites on its activation through binding with RACK. Therefore, RACK represents a part of PKC signaling cascade. Its transcriptional enhancement in the brains of SD- and DP-conditioned larvae indicates that this cascade might be activated under these conditions. Although the detailed function of RACK in the brain of M. brassicae larvae exposed to SD length or fed Dopa remains unknown, signal transduction through PKC could contribute to transduce these stimuli into the diapause inductive program.

In conclusion, the diapause-inducing stimuli such as SD-condition and feeding Dopa elevated transcriptional 


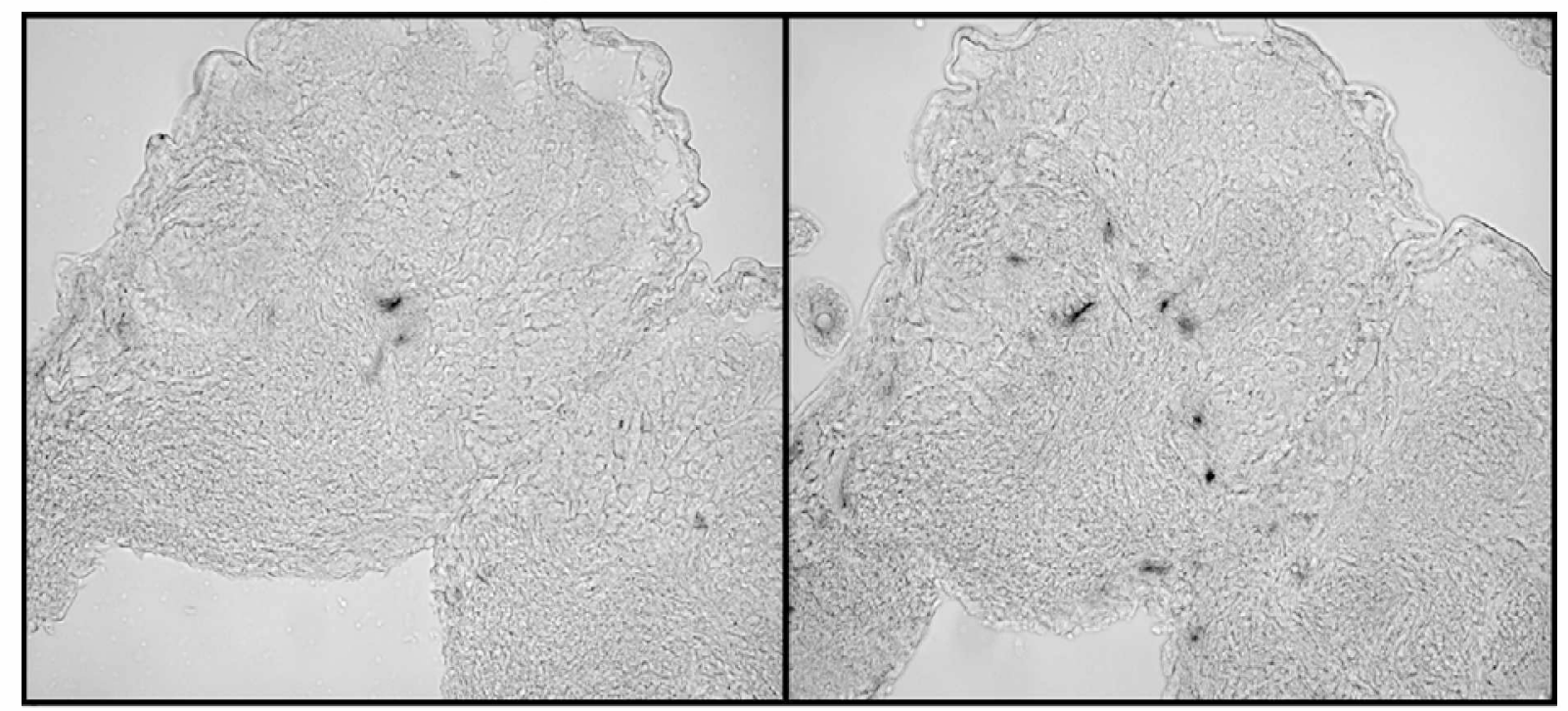

\section{(C) Control}

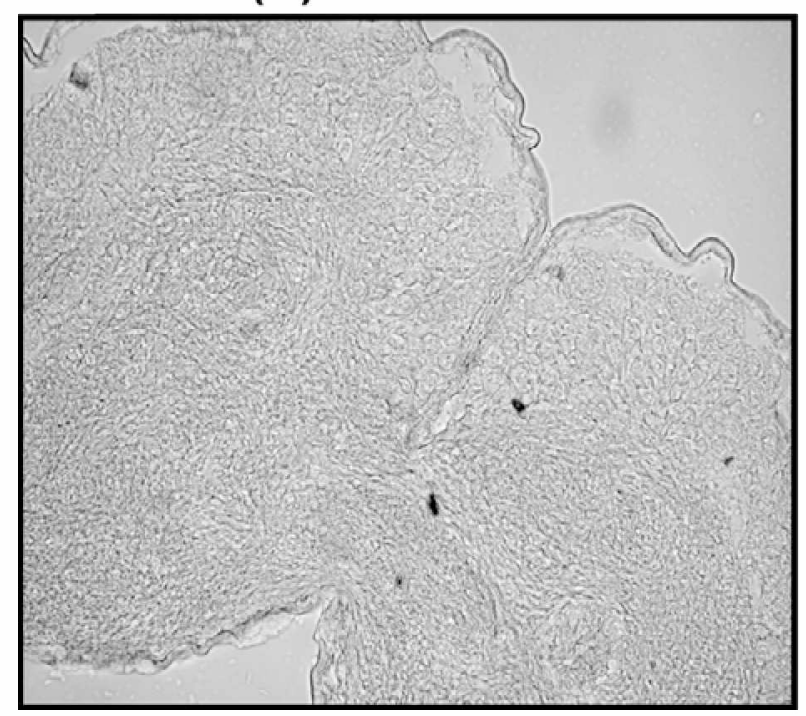

levels of several genes in the $M$. brassicae larval brains. Using the subtractive hybridization technique, we identified a total 16 genes whose expression was enhanced by the SD- or Dopa-treatment. Among them, the RACK (receptor for activated protein kinase C) gene is the most up-regulated under both conditions. The RACK mRNA increased in the larval brain within one day of feeding Dopa and maintained the increased levels at least for 3 days. The RACK gene expression was also enhanced in the brains of larvae within 2 days of transfer from LD to SD. Therefore, it is expected that these diapause-inducing stimuli could be transduced into the diapause program in the brain through protein kinase $\mathrm{C}$.

\section{REFERENCES}

Chomczynski P. \& SACChI N. 1987: Single step method of RNA isolation by acid guanidium thiocyanate-phenol-chloroform extraction. Anal. Biochem. 162: 156-159.

Denlinger D.L. 1985: Hormonal control of diapause. In: Kerkut G.A. \& Gilbert L.I. (eds): Comprehensive Insect Physiology,
Fig. 5. Cellular localization of expression of the RACK mRNA in the brain sections of $M$. brassicae larvae kept under LD and SD. A - brain section of day 3 penultimate instar larva kept under LD; B - brain section of day 3 penultimate instar larva kept under SD for 3 days after transfer from LD; C - brain section of the same conditioned larva hybridized with the sense probe.
Biochemistry and Pharmacology, Vol. 8. Pergamon Press, New York, pp. 353-412.

HAYAKAWA Y. \& NoGUCH H. 1998: Growth-blocking peptide expressed in the insect nervous system, cloning and functional characterization. Eur. J. Biochem. 253: 810-816.

HaYaKawa Y. \& OhnishI A. 1998: Cell growth activity of growth-blocking peptide. Biochem. Biophys. Res. Commun. 250: 194-199.

HaYaKawa Y., Ohnishi A. \& Endo Y. 1998: Mechanism of parasitism-induced elevation of haemolymph growthblocking peptide levels in host insect larvae (Pseudaletia separata). J. Insect Physiol. 44: 859-866.

Hayakawa Y., Ohnishi A., Yamanaka A., Izumi S. \& Tomino S. 1995: Molecular cloning and characterization of cDNA for insect biogenic peptide, growth-blocking peptide. FEBS Letters 376: 185-189.

Kmura Y. \& MASAKI S. 1992: Effect of light period on darktime measurement for diapause induction in Mamestra brassicae. J. Insect Physiol. 38: 681-686.

Kmura Y. \& MASAKI S. 1993: Hourglass and oscillator expressions of photoperiodic diapause response in the cabbage moth Mamestra brassicae. Physiol. Entomol. 18: 240-246. 
Kraft A.S. \& Anderson W.B. 1983: Phorbol esters increase the amount of $\mathrm{Ca}_{2}{ }^{+}$, phospholipid-dependent protein kinase associated with plasma membrane. Nature 301: 621-623.

Meldrum E., Parker P.J. \& Carozzi A. 1991: The PtdIns-PlC superfamily and signal transduction. Biochem. Biophys. Acta 1092: 49-71.

Menssen A. \& Hermering H. 2002: Characterization of the c-MYC-regulated transcriptome by SAGE: identification and analysis of c-MYC target genes. Proc. Natl. Acad. Sci. USA 99: 6274-6279.

NishizuKa Y. 1984: The role of protein kinase C in cell surface signal transduction and tumour promotion. Nature 308: 693-698.

NisHzuKa Y. 1988: The molecular heterogeneity of protein kinase $\mathrm{C}$ and its implications for cellular regulation. Nature 334: $661-665$.
Noguchi H. \& Hayakawa Y. 1997: Role of dopamine at the onset of pupal diapause in the cabbage armyworm Mamestra brassicae. FEBS Letters 413: 157-161.

Noguchi H. \& HaYakawa Y. 2001: Dopamine is a key factor for the induction of egg diapause of the silkworm, Bombyx mori. Eur. J. Biochem. 268: 774-780.

Tanaka K., Matsumoto H. \& Hayarawa Y. 2002: Detailed characterization of polydnavirus immunoevasive proteins in an endoparasitoid wasp. Eur. J. Biochem. 269: 2557-2566.

Tauz D. \& Pfeifle C. 1989: A non-radioactive in situ hybridization method for the localization of specific RNAs in Drosophila embryos reveals translational control of the segmentation gene hunchback. Chromosoma 98: 81-85.

Received August 21, 2002; revised February 3, 2003; accepted March 17, 2003 